\title{
Prediction of Nonlinear Dynamic Characteristics and Stability of Aerodynamic Bearing
}

\author{
Chenhui Jia ${ }^{1, a^{*}}$, Xiao Meng ${ }^{2, b}$ and Ming QIU ${ }^{3, c}$ \\ ${ }^{1,2,3}$ Affiliation: School of Mechatronics Engineering, Henan University of Science and Technology, \\ Luoyang, Henan, China; \\ axjiachenhui@163.com, bgymengxiao@163.com, cqiuming69@126.com
}

*Address correspondence to this author at Xiyuan Road 48, Box 62, Luoyang, Henan, China, 471003; Tel: 0086-13523798226; Email:xjiachenhui@163.com

\begin{abstract}
Keywords: Aerodynamic bearing; static performance; Dynamic stiffness coefficients; Dynamic damping coefficients; Nonlinear dynamic characteristics.
\end{abstract}

Abstract. In order to research the transient-state flow field characteristics of spherical spiral groove aerodynamic bearings, the bearing's 3D micro gas film mathematical model was established, and analyzed the 3D gas film pressure field of the bearing based on the FLUENT software of fluid dynamics, and obtained the bearing structure parameters and operation parameters of the maximum bearing load capacity. Based on the maximum bearing capacity, the effects of different speeds and eccentricity ratio on dynamic characteristic coefficients were studied, and to explore the gas bearing's transient nonlinear dynamic behavior. The axis orbit of the bearing in different speeds is simulated and the stability of bearing is researched. The results show that based on the maximum bearing capacity, a reasonable choice of the bearing speed and eccentricity contributes to improve the dynamic characteristics, and the stability of rotor-bearing system. The paper provides a theoretical prediction to improve the stability of gas bearing in complex environment.

\section{Introduction}

Due to the high rotation speed of aerodynamic bearings that can reach several hundreds of thousands revolutions per minute, the gas film pressure changes presented a very complicated nonlinear stochastic process $[1,2,3,4]$. When subjected to the influence of external factors, the rotor will perform random movement with the change of load in the center of axis. The dynamic characteristic coefficients reflect to the internal relations between the displacement and velocity when the rotor is subjected to external loads variation [5,6], and it can be characterized by the dynamic stiffness and dynamic damping of the micro film and analyzed the rotor's unbalance response, critical speed \& stability [7, 8]. Based on the axis orbit, the working condition of the gas bearing can be obtained, and the minimum gas film thickness and the rotation accuracy of the bearing can be obtained, and the stability of the bearing is also determined $[9,10,11,12]$.

In order to research the transient-state flow field characteristics of spherical spiral groove aerodynamic bearings, the bearing's 3D micro gas film model was established, and then analyzed the influence of the bearing dimensionless eccentricity, dimensionless groove width ratio, dimensionless groove depth ratio, rotation speed variations to bearing capacity, and obtained the bearing structure parameters and operation parameters of the maximum bearing capacity. Based on the maximum bearing capacity, the effects of different speeds and eccentricity ratio on dynamic characteristic coefficients were studied, and analyzed the effects of different speeds and eccentricity ratio on stability. The axis orbit of the bearing in different speeds was simulated and the stability of bearing was researched.

\section{Mathematical modeling}

The spherical spiral groove aerodynamic bearing is mainly composed of rotor and stator, the surface of the rotor is machined a certain number of spiral grooves, and the spiral groove consists of ridge and 
groove, the larger part of gap called groove and the smaller called ridge, the spherical spiral groove aerodynamic bearing section sketch is illustrated in Fig. 1. As the rotor rotates relative to the stator at a high speed, the spiral groove not only forms the staircase effect on bearing, but also increases the pump bearing gas effect, which well promotes aerodynamic bearing hydrodynamic effect.

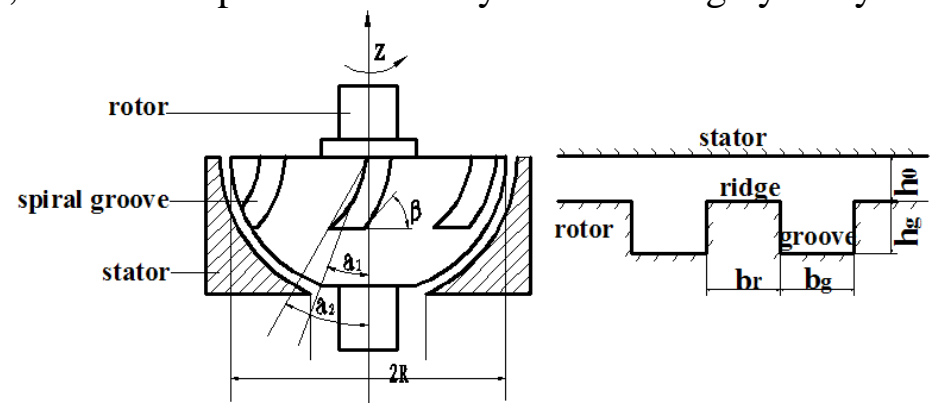

Fig. 1. Schematic diagram of spherical spiral groove aerodynamic bearing

$\omega$ is rotational speed, $b_{r}$ is ridge width, $b_{g}$ is groove width, $h_{g}$ is film thickness at a groove, angle $\alpha_{1}=25^{\circ}, \alpha_{2}=47^{\circ}$, groove number $N_{g}=5$, spiral angle $\beta=70^{\circ}$, Bearing radius $R=4.8 \mathrm{~mm}$; gas film clearance $h_{0}=10 \mu \mathrm{m}$; gas viscosity $\mu=1.833 \times 10^{-5} \mathrm{pas}$; atmospheric pressure $p_{0}=1.013 \times 10^{5} \mathrm{pa}$; dimensionless eccentricity $\varepsilon=0.4$; dimensionless groove width ratio $\bar{b}=b_{r} /\left(b_{r}+b_{g}\right)=0.5$; dimensionless groove depth ratio $\bar{h}=\left(h_{0}+b_{g}\right) / h_{0}=3.6$.

\section{Finite Element Model}

The model of the spiral line is established by space spherical coordinates. The 3D gas film model of the spherical spiral groove aerodynamic bearing is established by the 3D model software Pro/E, the 3D model of the gas film is shown in Fig. 2.

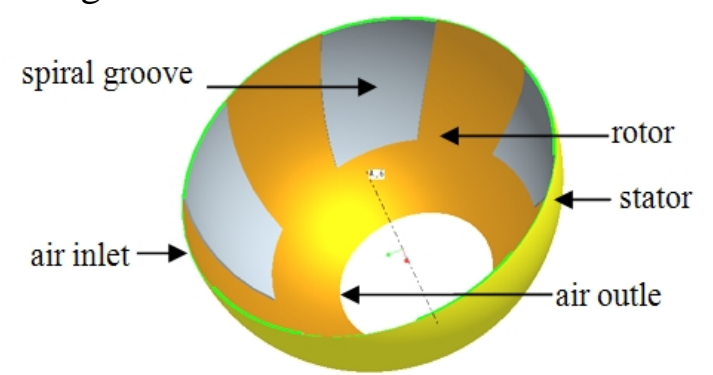

Fig. 2. The 3D model of the gas film of spherical spiral groove aerodynamic bearing

\section{Steady-state characteristic factors}

The 3D gas film model of the bearing is established by the 3D model software Pro/E, using the former processing software ICEM software to grid the gas film bearing model, and three boundary condition of air inlet, air outlet and wall boundary conditions were set. The mass conservation equation and the momentum conservation equation were dispersed by the finite volume method, and the pressure difference format was linear differential. Other discrete equations were the second-order difference format, and the method of pressure-velocity coupling was the SIMPLE algorithm. The gas film flow field were simulated, and obtained gas film pressure distribution contours and radial $\&$ axial bearing capacity.

The load capacity is an important index to reflect bearing performance. This paper mainly analyses the effect to the load capacity with the change of operational parameter of rotation speed $N$ and structural parameters of groove width ratio $\bar{b}$, groove depth ratio $\bar{h}$, eccentricity $\varepsilon$.

Fig. 3-1 shows the load capacity curve of the different rotation speed is consistent with the change of groove width ratio $\bar{b}$. With the increase of the rotation speed $N$, the load capacity of the bearing is 
better. The bearing capacity increases with the increase of the ratio of groove width $\bar{b}$. When $\bar{b}=0.5$, the bearing capacity of three curves is up to the maximum value.

Fig. 3-2 shows the load capacity curve of the different rotation speed is consistent with the change of the groove depth ratio $\bar{h}$, and with the increase of the rotation speed $N$, the capacity of the bearing is better. The bearing capacity increases with the increase of the ratio of the groove depth ratio $\bar{h}$. When the groove depth ratio is 3.6, the load capacity is the most suitable for the aerodynamic bearing, and then bearing capacity decreases with the increase of groove depth ratio.

Fig. 3-3 shows the load capacity curve of the different rotation speed is consistent with the change of the eccentricity $\varepsilon$. Along with the increase of the eccentricity $\varepsilon$, the load capacity is increasing and the hydrodynamic effect is more obvious.

When $\varepsilon=0.4, \bar{b}=0.5, \bar{h}=3.6$, the bearing capacity of three curves is up to the maximum value. In this paper, the analysis of nonlinear dynamic characteristics and stability of the bearing would be used in these parameters.

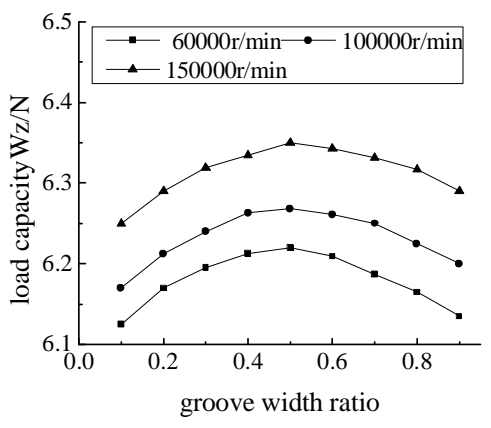

Fig. 3-1 Variation of $W_{Z}$ with $\bar{b}$

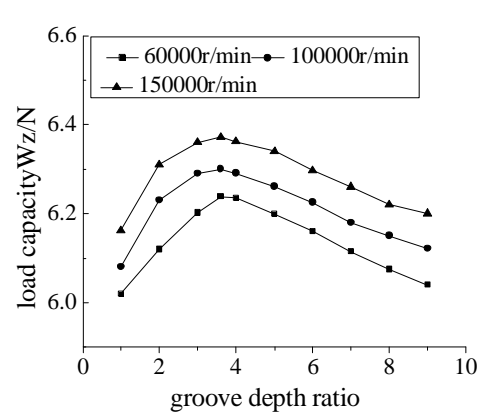

Fig. 3-2. Variation of $W_{Z}$ with $\bar{h}$

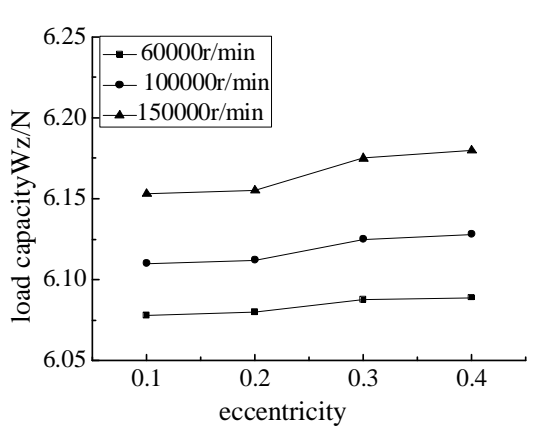

Fig.3-3. Variation of $W_{Z}$ with $\varepsilon$

\section{Analysis of dynamic characteristic coefficients of bearing}

\section{The differential calculation model of stiffness and damping coefficients}

When the rotor is subjected to displacement and velocity fluctuation in static equilibrium position, and the gas film pressure changes is a very complicated nonlinear stochastic process. If the rotor deviated from the steady state position $O_{1}$ in the $\xi, \phi, z$ directions, force analysis as shown in Fig. 4. The gas pressure of the steady state position can be expressed as: $p_{0}=\left(\xi, \phi, z ; e_{0}, \theta_{0}, z_{0} ; 0,0,0\right)$, transient pressure distribution: $p=p(\xi, \phi, z ; e, \theta, z ; \&, e \notin, \&)$.

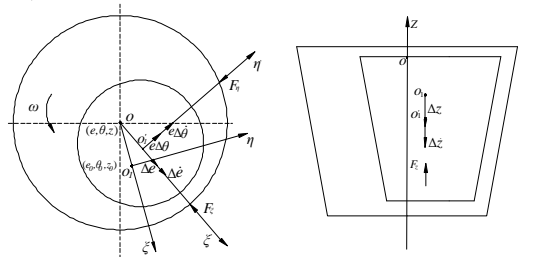

Fig. 4. Force analysis

\section{The Calculation method of stiffness coefficients}

When rotor has a small perturbation displacement in the horizontal direction, the perturbation displacement in vertical direction and the perturbation velocity in two other directions of the gas film force are zero, and then the gas film force increment can be expressed as Eq.1:

$$
\left\{\begin{array}{l}
\Delta F_{e}=k_{e e} e \\
\Delta F_{\theta}=k_{\theta e} e \\
\Delta F_{z}=k_{z e} e
\end{array}\right.
$$

First, the gas film force increment $\Delta F_{\mathrm{e}}, \Delta F_{\theta}$ and $\Delta F_{\mathrm{z}}$ were calculated when the rotor was deviated before and after in the horizontal and vertical direction. The gas film stiffness coefficients $k_{\mathrm{ee}}, k_{\theta \mathrm{e}}, k_{\mathrm{ze}}$ and $k_{\mathrm{e} \theta}, k_{\theta \theta}, k_{\mathrm{ze}}$ and $k_{\mathrm{ez}}, k_{\theta \mathrm{z}}, k_{\mathrm{zz}}$ can be obtained by rotor's tiny displacement perturbation $e / h_{0}, \theta / h_{0}$ and $z / h_{0}\left(e / h_{0}, \theta / h_{0}\right.$ and $z / h_{0}$ were $\left.0.001 \mathrm{~mm}\right)$ in $\mathrm{x}, \mathrm{y}, \mathrm{z}$ direction. 
Fig. 5 shows the variation law of the calculated values of the dynamic stiffness coefficients with the speed $(\mathrm{N}=80000,120000 \mathrm{r} / \mathrm{min})$ and the eccentricity $(\varepsilon=0.1,0.2,0.3$, and 0.4$)$ :

(1) The main stiffness coefficients $k_{\mathrm{ee}}, k_{\theta \theta}, k_{\mathrm{zz}}$ are linearly increased with the increase of speed and eccentricity, and the cross-coupled stiffness coefficients also present a tendency of increasing. It was found that the main stiffness coefficients increased obviously than the cross-coupled stiffness coefficients, and which revealed that the higher of the rotation speed, the more obvious of hydrodynamic effect of the aerodynamic bearings.

(2) In addition to the cross-coupled stiffness coefficients $k_{\mathrm{e} \theta} 、 k_{\mathrm{ez}}$, the other cross-coupled stiffness coefficients of aerodynamic bearing increased obviously with the increase of eccentricity $\varepsilon$.

(3) As the main support directions $\xi, z$ of the loaf capacity, the values of the direct stiffness coefficients $k_{\mathrm{ee}}, k_{\mathrm{zz}}$ are larger. The $\phi$ is the non-main support direction, so the value of stiffness coefficient $k_{\theta \theta}$ is relatively small. In general, the cross-coupled stiffness coefficient $k_{\mathrm{e} \theta}, k_{\theta \mathrm{e}}, k_{\mathrm{ze}}$ and $k_{\theta \mathrm{z}}$ in the non-main support direction are a little less than cross-coupled stiffness coefficient $k_{\text {ez }}$ and $k_{\text {ze }}$.
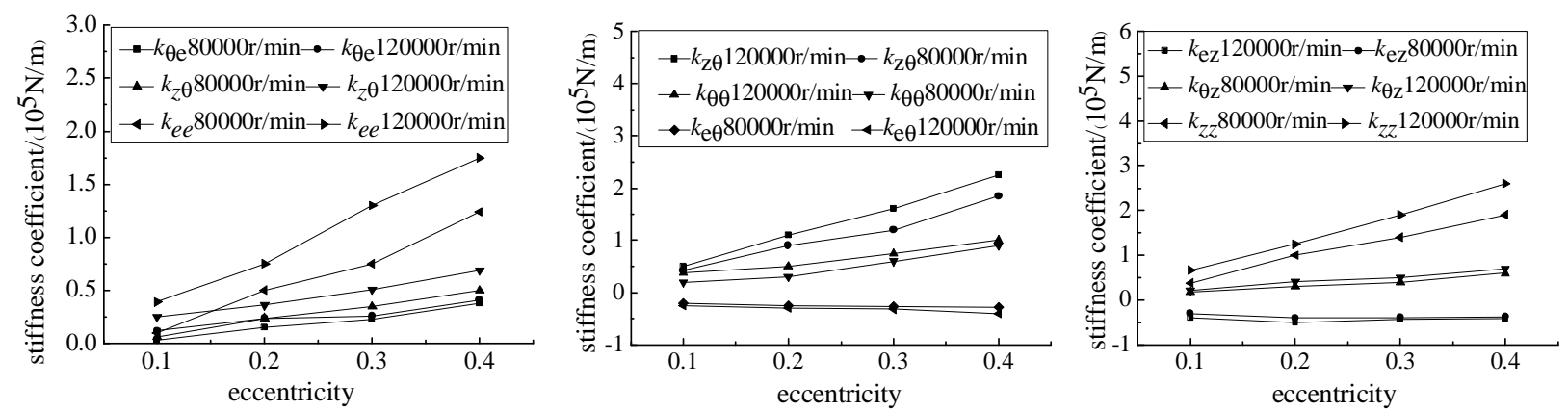

Fig. 5. Variation law of the calculated values of dynamic stiffness coefficients with $N$ and $\varepsilon$

\section{The Calculation method of damping coefficients}

When the rotor has a small perturbation velocity in the horizontal direction, the perturbation velocity in the vertical direction and perturbation displacement in other two directions of gas film force are zero, and then the gas film force increment can be expressed as Eq.2:

$$
\left\{\begin{array}{l}
\Delta F_{e}=b_{e e} \& \\
\Delta F_{\theta}=b_{\theta e} \& \\
\Delta F_{z}=b_{z e} \&
\end{array}\right.
$$

First, the gas film force increment $\Delta F_{\mathrm{e}}, \Delta F_{\theta}$ and $\Delta F_{\mathrm{z}}$ were calculated when the rotor was deviated before and after in the horizontal and vertical direction. The gas film stiffness coefficients $b_{e e}, b_{\theta e}, b_{z e}$ and $b_{e \theta}, b_{\theta \theta}, b_{z \theta}$ and $b_{e z}, b_{\theta z}, b_{z z}$ can be obtained by rotor's tiny displacement perturbation $\left(\& N h_{0}, \& N h_{0}\right.$ and $\xi / N h_{0}$ were $0.001 \mathrm{~mm}$ )in $x, y, z$ direction.
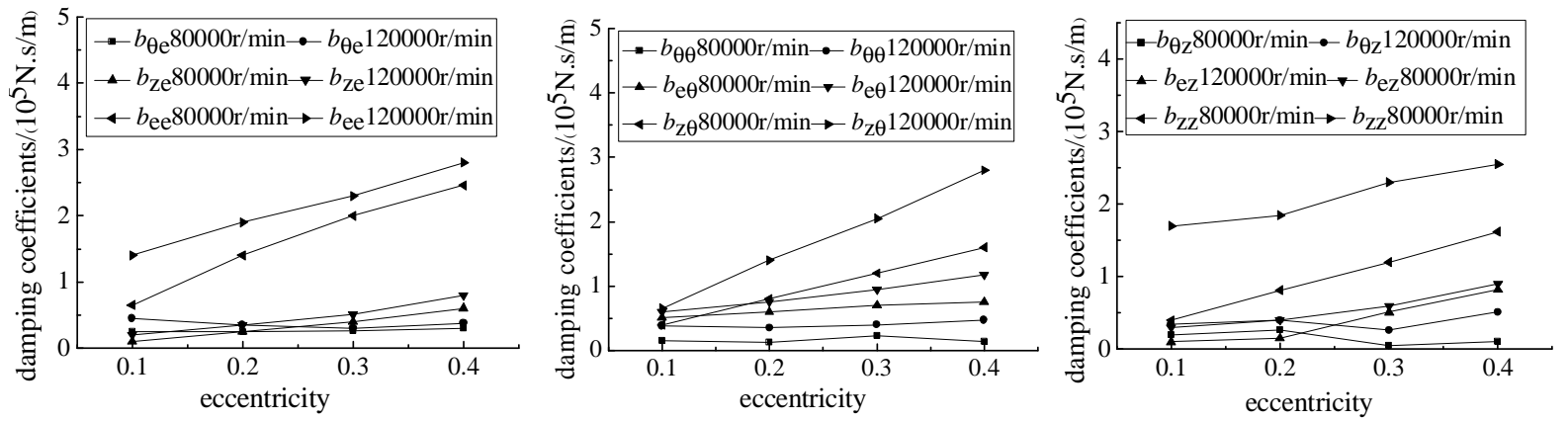

Fig. 6. Variation law of the calculated values of dynamic damping coefficients with $N$ and $\varepsilon$

Fig. 6 shows the variation law of the calculated values of the dynamic damping coefficients with the speed $(N=80000,120000 \mathrm{r} / \mathrm{min})$ and the eccentricity $(\varepsilon=0.1,0.2,0.3,0.4)$ : 
The main damping coefficients $b_{\mathrm{ee}}, b_{\theta \theta}, b_{\mathrm{zz}}$ are increased with the increase of the speed and eccentricity, and which reveals that the higher of the rotation speed, the more obvious of hydrodynamic effect of the aerodynamic bearings.

As the main support directions $\xi$, $z$ of the load capacity, the value of the damping coefficients $b_{\text {ee }}$, $b_{\mathrm{zz}}$ are large. While the value of direct damping coefficient is small as the $\phi$ is the non-main support direction. The cross-coupled damping coefficients $b_{\mathrm{e} \theta}, b_{\theta \mathrm{e}}, b_{\mathrm{z} \theta}$ and $b_{\theta \mathrm{z}}$ related to the non-main support directions are a little less than the cross-coupled damping coefficients $b_{\text {ez }}$ and $b_{\text {ze }}$.

\section{Analysis of stability}

The Jeffcott rotor system is supported by two aerodynamic bearings which are taken as the research object, and the bearing-rotor system is shown in Fig 7.

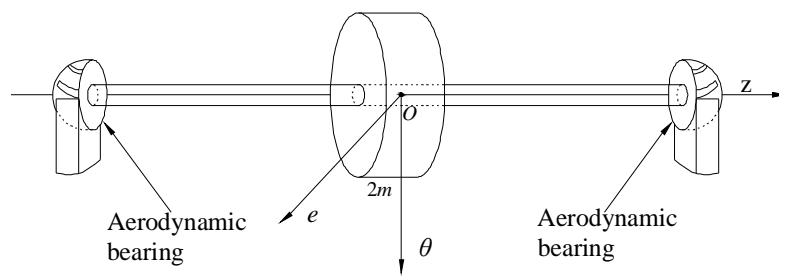

Fig. 7. The Schematic diagram of the Jeffcott rotor model

The transient flow field of the bearing-rotor system was solved based on the method of gas-solid coupling. The gas film force can be solved by FLUENT, the acceleration, velocity and displacement can be obtained by equation $(3,4,5)$. The axis orbit was simulated and analyzed the stability of rotor-bearing system.

$$
\begin{aligned}
& \left\{\begin{array}{l}
\frac{f_{e e}-f_{e}}{m} \\
\frac{f_{e \theta}-f_{\theta}+m g}{m}
\end{array}\right. \\
& \left\{\begin{array}{l}
\&=\&+t \\
\theta=\alpha_{0}^{\alpha}+t
\end{array}\right.
\end{aligned}
$$

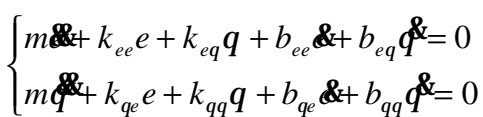

Fig. 7 shows axis orbit of the aerodynamic bearing in the speed $60000 \mathrm{r} / \mathrm{min}, 100000 \mathrm{r} / \mathrm{min}$, the mass of rotor on this bearing is $0.09 \mathrm{~kg}$, mass eccentricity $e_{x}=e_{y}=1.0 \times 10^{-6} \mathrm{~m}$.

Fig. 7 shows with the speed increased, the stability margin of the bearing-rotor system is decreased. When the speed reached $100000 \mathrm{r} / \mathrm{min}$, the axis orbit is easily disturbed, and then bearing rotor system is prone to instability phenomenon.

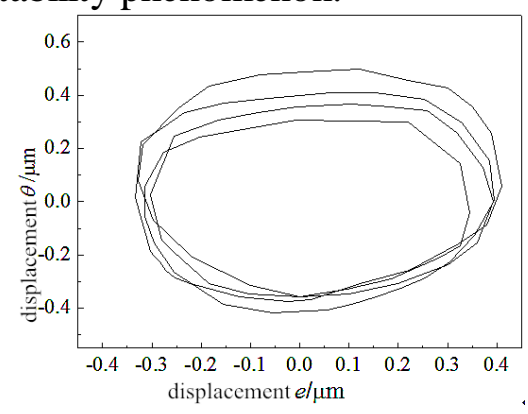

(a) $N=60000 \mathrm{r} / \mathrm{min}$

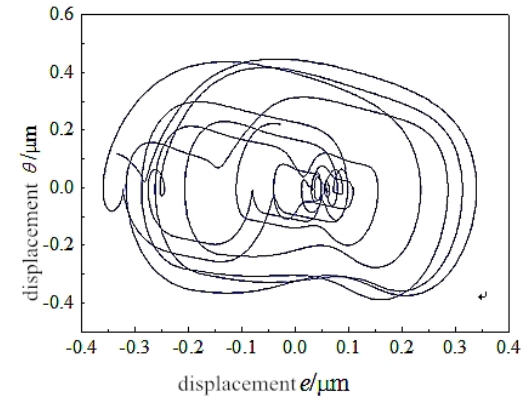

(b) $N=100000 \mathrm{r} / \mathrm{min}$

Fig. 8. The charts of axis orbit in different speed 


\section{Conclusions}

(1) Under different rotation speed, the bearing structural parameters with groove width ratio, groove depth ratio, and eccentricity have more obvious effect on the bearing capacity.

(2) The increase of the bearing's speed contributes to improve the dynamic characteristic coefficients, while the system stability approaches the critical state. The system stability in the low speed range is better than the stability in the high speed range.

(3) With the increase of the eccentricity, the stiffness coefficients increases obviously, while the damping coefficients increase easing up. The system stability approaches the steady state with the increase of the eccentricity.

(4) The influence of the dynamic characteristic coefficients on the stability of the bearing-rotor system depends on the comprehensive effect of stiffness and damping. The increased of the bearing's speed and eccentricity contributes to improve the dynamic damping coefficients of bearings. Meanwhile, the dynamic damping coefficients have a certain inhibiting effect on the axis orbit. It can consume the whirl energy and improve the stability of the bearing-rotor system. But the stability of the bearing-rotor system will approaches the critical state with the increase of the speed constantly, which is a factor for the system instability. The eccentricity contributes to inhibit the whirling, but the large eccentricity can generate collision and friction to damage the bearing. Therefore, a reasonable choice of design parameters contributes to improve the bearing's comprehensive properties.

\section{Acknowledgement}

This work is supported by National Natural Science Foundation of China (Grant no: 51475142), and Program for Innovative Research Team (in Science and Technology) in University of Henan Province (Grant no: 13IRTSTHN025), and the Education Department Henan Province Science and Technology Research Projects (Grant no: 13A460251).

\section{References}

[1] Yang Pan, Zhu Keqin, Wang Xiaoli. On the non-linear stability of self-acting gas journal bearings [J]. Tribology International (0301-679X), 2009, 42(1): 71-76.

[2] Fredirk Sahlin. CFD-analysis of Hydrodynamic Lubrication of Textured Surfaces [J]. The Inclined Groove Bearing, 2003.

[3] A. Jourak. CFD Analysis of a Journal Bearing with a Microgroove on the Shaft [C]. The 19th International Symposium on Transport Phenomena, 2008: 17-20.

[4] Al-Bender F. On the Modelling of the Dynamic Characteristics of Aerostatic Bearing Films: From Stability Analysis to Active Compensation [J]. Precision Engineering, 2009, 33(2): 117-126.

[5] Maru PE, Star M. Rigorous Justification of the Reynolds Equations for Gas Lubrication [J]. Comptes Rendus-Mecanique, 2005, 333(7): 534-541.

[6] Turchak LI, Shidlovskii VP. Theoretical and Numerical Investigation of Gas Lubrication Processes on the Basis of Aerodynamic Equations [J]. Fluid Dynamics, 2001, 36(5): 691-700.

[7] Wang C C, Chen C K. Bifurcation Analysis of Self-acting Gas Journal Bearing [J]. ASME, Journal of Tribology, 2001, 123(44): 755-767.

[8] Belforte G, Raparelli T, Viktorov, et al. A Theoretical Study of a High Speed Rotor Supported by Air Bearings Mounted on O-Rings [C]. Fifth International Conference on Tribology, 2006, 1-9.

[9] Bou-Sa B, Grau G, Iordanoff I. On Nonlinear Rotor Dynamic Effects of Aerodynamic Bearings with Simple Flexible Rotors [J]. Journal of Engineering for Gas Turbines and Power, 2008, 130(1): 0125031-0125039. 
[10] Kim D, Lee S, Bryant M D, et al. Hydrodynamic Performance of Gas Microbearing[J]. Journal of Tribology, 2004, 126: 711. 